\title{
MODIFICATION OF RETROGRADE DEGENERATION IN TRANSECTED SPINAL AXONS OF THE LAMPREY BY APPLIED DC CURRENT $^{1}$
}

\author{
ERNESTO ROEDERER, NELSON H. GOLDBERG, ${ }^{2}$ AND MELVIN J. COHEN ${ }^{3}$ \\ Department of Biology, Yale University, New Haven, Connecticut 06511
}

Received June 9, 1982; Accepted July 27, 1982

\begin{abstract}
In the spinal cord of the lamprey, regeneration of giant reticulospinal axons occurs following transection. We show that partial degeneration of the proximal axonal segment, or "die-back," also occurs following spinal transection and it precedes regenerative outgrowth. The die-back during the first 5 days post-transection is reduced significantly by application of a $10-\mu \mathrm{A} \mathrm{DC}$ current across the site of transection, with the cathode distal to the lesion. Reversing the polarity of the applied current (cathode proximal to the lesion) increases the extent of axonal die-back relative to the sham-treated controls. Following spinal transection, saline-filled wick electrodes were implanted in the body musculature on either side of the lesion. Electrically treated animals received current across the lesion for 5 days, while the sham-treated controls received no current. After 5 days, several giant axons in each preparation were injected intracellularly in the spinal cord with the dye Lucifer Yellow. The extent of axonal die-back in the proximal cord stump was determined in the filled fibers by measuring the distance of the axon end from the site of lesion. The mean distances of axonal dieback were as follows: controls, $1750 \mu \mathrm{m} \pm 45$ SEM; cathode-distal, $740 \mu \mathrm{m} \pm 33$ SEM; cathodeproximal, $2820 \mu \mathrm{m} \pm 60 \mathrm{SEM}$. These differences between the treatment groups proved to be significant using the Wilcoxon rank sum test. We propose that die-back is caused by the entry of cations driven into the cut surface of the cord by the endogenous injury current. The applied DC current interacts with the endogenous current of injury to either decrease or increase the flow of cations into the cord, depending on the direction of applied current flow across the lesion. This in turn causes a corresponding reduction or enhancement of the axonal die-back.
\end{abstract}

The complex shape of a differentiated neuron may result from a continuous dynamic interplay between the breakdown and assembly of the cytoskeletal elements (Lasek, 1981). Experimental axotomy evokes a sequence of plastic alterations in nerve cell form involving both the breakdown and the growth of neurites still attached to the soma. We have therefore used this procedure to study axon degeneration and growth in giant reticulospinal neurons within the brain and spinal cord of a primitive vertebrate, the lamprey Petromyzon marinus. We examined in particular the early degeneration of the ends

\footnotetext{
${ }^{1}$ We thank Mary McHale and Shawn Lockery for excellent technical assistance. This work was supported by National Institutes of Health Spinal Trauma Center Grant 2P50 NS 10174-09, National Institutes of Health Biomedical Research Support Grant 2-SO7-RR07015, and the Charles Oshe Fund of Yale University.

${ }^{2}$ Present address: Department of Surgery (Plastic). University of Maryland Hospital, 22 South Greene Street, Baltimore, MD 21201.

${ }^{3}$ To whom correspondence should be addressed at Department of Biology, Yale University, P. O. Box 6666, New Haven, CT 06511.
}

of severed axons in the proximal spinal cord stump that precedes their regenerative outgrowth.

It has long been known that an initial axonal response to spinal cord injury consists of a retrograde degeneration in the proximal axonal stump that proceeds from the site of lesion toward the brain for several millimeters. Cajal (1928) described an early retrograde "traumatic degeneration" that occurred in a progressive manner from the site of lesion for 1 to $2 \mathrm{~mm}$ into the proximal cord stump. He differentiated this process from the Wallerian degeneration of axon segments detached from their cell bodies. He suggested that this limited, progressive fragmentation of the proximal axon segment occurred by the formation of swollen retraction buds and their eventual detachment and dissolution.

A variety of other mechanisms have been proposed more recently to account for this early progressive retrograde degeneration or "die-back" of spinal axons following injury. These include an active extrusion of axoplasm driven from the cut end by axoplasmic flow (Kao 
et al., 1977). 'The swelling of the retraction bud also has been attributed to the accumulation of axoplasm at the cut ends due to orthograde axoplasmic flow (Friede and Martinez, 1970). Lysosomes are prevalent in the retraction bud and their lytic enzymes are suggested as the cause of the degradation of the tip of the injured axon that leads to the formation of microcysts. These microcysts then coalesce to result in cavitation of the proximal and distal stumps seen following injury to the mammalian spinal cord (Kao and Chang, 1977).

Previously we have found in the lamprey that a DC current applied across the site of a spinal cord transection for 5 days causes an enhancement in the regeneration of giant reticulospinal axons observed 40 to 60 days after the lesion (Borgens et al., 1981). In the present study we ask whether this enhanced spinal cord regeneration induced by applying DC current might be due to a modification of the early die-back process. We also sought to determine the effect of the direction of current flow across the lesion on the extent of die-back. We therefore applied $10 \mu \mathrm{A}$ of $\mathrm{DC}$ current across the site of transection for 5 days and then examined the extent of die-back in single giant reticulospinal axons at that time. We find that applied current across the lesion can significantly modify the extent of die-back in severed axons of the proximal cord stump. We feel that this may play a role in enhancing spinal axon regeneration following application of DC current across the site of injury in the transected spinal cord.

\section{Materials and Methods}

Amocoete larvae of the lamprey Petromyzon marinus 8 to $10 \mathrm{~cm}$ in length were obtained from fresh water streams in Maine. They were kept in the laboratory at $15^{\circ} \mathrm{C}$ for as long as 3 months in large aquaria with aerated well water and a substrate of sand. No additional food was provided other than that possibly present in well water.

Surgical procedure and electrical stimulation. All animals were anesthetized in a $0.033 \%$ solution of benzocaine in lamprey saline (Wickelgren, 1977). Under a stereo dissecting microscope a dorsal transverse incision, approximately $7 \mathrm{~mm}$ wide, was made $1 \mathrm{~cm}$ caudal to the last gill slit. This incision cut through the dorsal surface down to the notocord and, therefore, completely transected the spinal cord. The free cut ends of the cord were clearly visible under the stereo microscope. Using a blunt glass rod, two longitudinal tunnels, each about $2 \mathrm{~cm}$ long, were made between the dorsal skin and the body musculature, one rostral and the other caudal to the lesion. A wick electrode was inserted into each tunnel running parallel to the underlying spinal cord for a distance of approximately $1 \mathrm{~cm}$ distal and $1 \mathrm{~cm}$ proximal to the cut (Fig. 1). The wick electrodes (Patter Products, Beaverton, OR) consisted of a Silastic tube ( $\mathrm{OD}=0.025$ in., ID $=0.012$ in.) containing a cotton wick, which was filled with lamprey saline prior to implantation in order to provide electrical continuity. The wounds then were closed using 8-0 nylon sutures (Ethicon Inc., Somerville, NJ). Immediately following closure of the wound the animal was placed in a container filled with aerated saline. The two exposed ends of the wick electrodes were

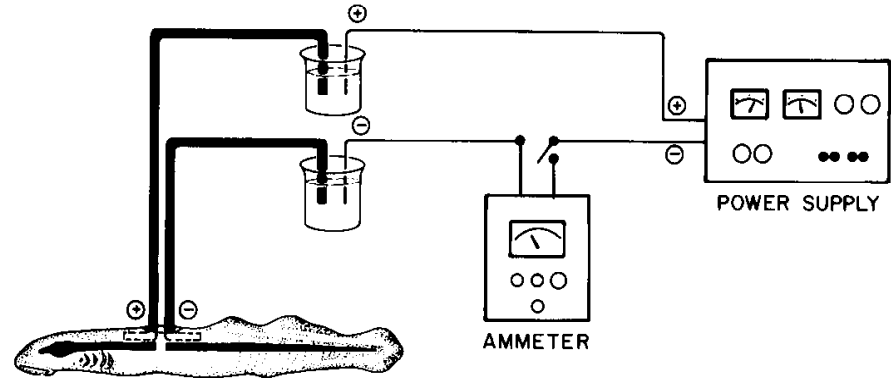

Figure 1. Diagram illustrating the method of applying electric current across the transected spinal cord with the cathode (-) placed distal to the site of transection. The current was delivered to the animal through wick electrodes filled with lamprey saline and connected to a DC power supply through saline bridges (small beakers). The power supply was connected to the saline bridge through chlorided silver wires. A microammeter was used daily to monitor the applied current flowing through each animal and to make any adjustments to ensure that this value was maintained at approximately $10 \mu \mathrm{A}$.

placed into saline wells which were connected to a Heathkit (model IP-17) DC power supply via chlorided silver leads (Fig. 1). Animals with implanted electrodes were divided into three groups: $(i)$ a "cathode-distal" treatment group in which current was passed across the transection with the cathode distal to the lesion; (ii) a "cathode-proximal" treatment group, in which current was applied with the cathode proximal to the lesion; and (iii) a "sham treatment" group (controls), in which electrodes were implanted but no current was applied. A Keithley model 602 microammeter was used to measure the applied current, and the voltage was adjusted to 60 to $70 \mathrm{DC}$ volts to deliver approximately $10 \mu \mathrm{A}$ across the lesion. The applied current was measured immediately after surgery, later that day (several hours postsurgery), and then was monitored daily during the 5-day treatment period. All animals were kept at 15 to $16^{\circ} \mathrm{C}$ throughout. During the first day after surgery, lampreys were maintained in saline to minimize any possible osmotic damage to surgically exposed tissues. Thereafter they were transferred to well water which was changed daily for the balance of the 5-day experimental period.

A total of 28 of 36 operated lampreys survived for 5 days after surgery and then were sacrificed and examined. The 8 animals lost were evenly distributed in the three treatment groups.

Physiology and anatomy. Five days after transection, the animal was anesthetized in a $0.033 \%$ solution of benzocaine in saline. The brain and the attached segment of the spinal cord proximal to the lesion were dissected completely free from the animal and pinned out on a clear Sylgard surface in a Petri dish filled with well oxygenated saline. The dura was removed and two pairs of silver wire electrodes were placed on the surface of the cord to stimulate and record extracellulary from the giant axons. The characteristic lateral location of the Mauthner axons and the ventromedial grouping of the Müller fibers (Fig. 2) could be observed in the isolated living spinal cord. This permitted the penetration of individual giant axons $1.5 \mathrm{~cm}$ proximal to the site of lesion using microelectrodes filled with the fluorescent dye Lucifer 


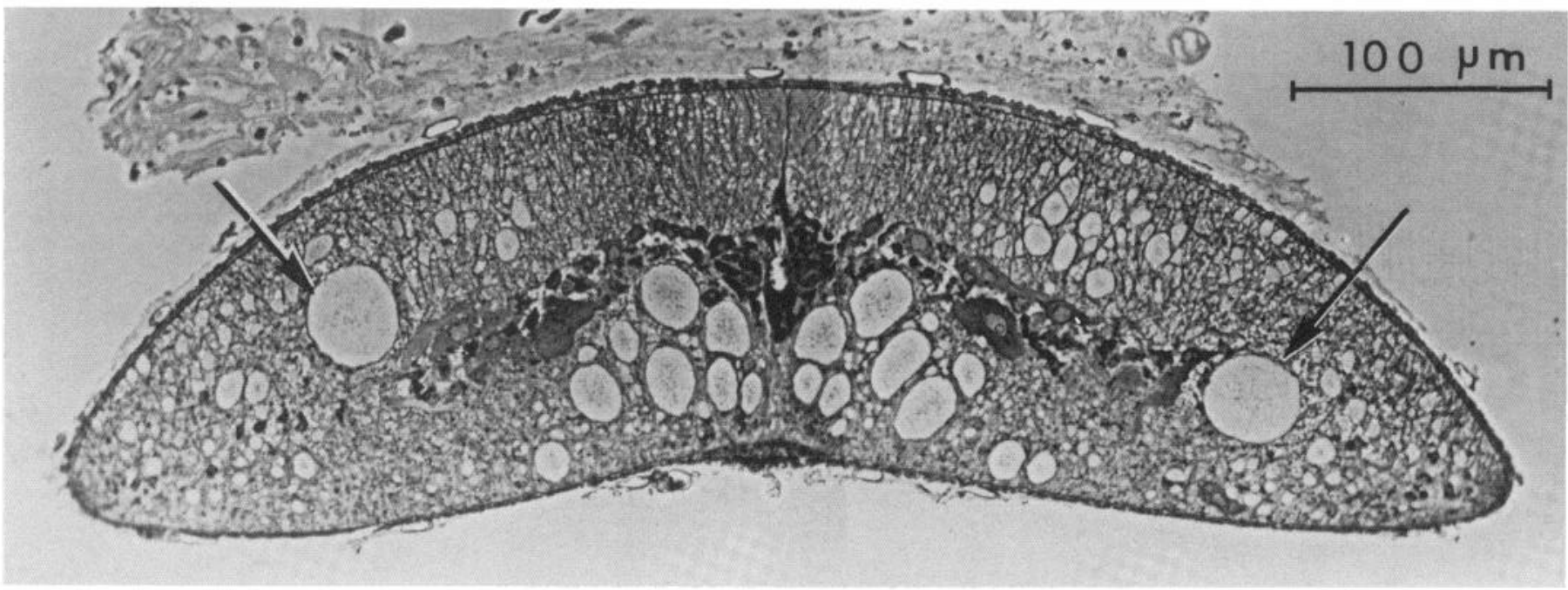

Figure 2. A 2- $\mu \mathrm{m}$ transverse section through a normal spinal cord made from a montage of two phase micrographs taken from a section stained with toluidine blue (dorsal surface upward). Two categories of giant reticulospinal axons are seen: the laterally located Mauthner axons are indicated by arrows; the large ventromedial profiles are axons of the Müller cells.

Yellow (Stewart, 1978). Hyperpolarizing current pulses of 500 msec duration and $20 \mathrm{nA}$ amplitude were passed at $1 \mathrm{~Hz}$ for $10 \mathrm{~min}$ in order to fill the axon with the fluorescent dye. The cords were allowed to stand for 2 to $3 \mathrm{hr}$ in saline to permit intracellular diffusion of the dye from the penetration point. They then were fixed, dehydrated, and cleared as previously described (Stewart, 1978). Finally, whole mounts of these cords were examined and photographed in transmission fluorescence optics on a Zeiss Universal microscope. Slides of a whole mount were projected to measure the distance of the end of each dye-filled axon from the site of lesion.

Tissue from some of the whole mounts was cleared in methyl salicylate and was prepared for sectioning as follows: a $0.5-\mathrm{cm}$ length of cord proximal to the lesion was placed in two changes of ethyl alcohol for $15 \mathrm{~min}$ each, then placed in a mixture of $50 \%$ propylene oxide and $50 \%$ Spurr's medium overnight. The tissue then was embedded in Spurr's medium, and $3-\mu \mathrm{m}$ sections were cut for examination with epifluorescent optics.

\section{Results}

We measured the distance between the ends of injected giant axons and the lesion 5 days following transection. The extent of this "die-back" was measured in the three treatment groups described above; i.e., a cathode-distal group, a cathode-proximal group, and the sham-treated controls. In the three treatment groups, all dye-filled axons had tips that were proximal to the site of transection; that is, all axons died back from the lesion. In any single preparation the extent to which different injected axons died back varied considerably. Despite this variability, it became apparent that there were differences in the extent of die-back in the three treatment groups. In the sham-treated controls, some axons ended near the lesion but others terminated several millimeters more proximal as shown in Figure 3 (top). On the other hand, in the cathode-distal group, axonal die-back from the lesion was not as extensive as in the controls. In several of these preparations, many axon ends were observed near the site of transection, as shown in Figure 3 (bottom). However, in the cathode-proximal group (Fig. 4a), the injected axons died back much further from the lesion than in either the controls or the cathode-distal preparations.

The nature of axonal die-back. The shape of the end of the dye-filled axons varied from a blunt termination to a fine tapered extension. There was often a mottled appearance in the dye density at the very end of the transected fibers and sometimes the stained material at the end was fragmented (e.g., Fig. $4 a$ ). The question thus arose whether these different dye-filled terminal configurations of severed axons indeed represented their true anatomical terminations.

To answer this point, we correlated the appearance of the filled, severed axons in the whole mounts with transverse $3-\mu \mathrm{m}$ sections of the same preparations examined in epifluorescence optics, as shown in Figure 4. Sections of such cords clearly show the location of each dye-filled axon. In addition, the background fluorescence in these sections revealed the outline of the cord and some of the internal structure, such as the central canal and profiles of other unfilled giant fibers. In the most proximal crosssection (Fig. $4 b$ ), the number and location of the bright fluorescent profiles correspond to the number and position of the filled fibers seen in the whole mount. Large axon profiles with a slight background fluorescence are unstained giant fibers. When following a dye-injected profile distally in successive transverse sections, the fluorescence may fragment (Fig. $4 c$ ) and, in more distal sections, the fluorescence is lost altogether (Fig. 4, $c, d$, and $e$ ). Regions of fluorescent fragments in the sections correlate with areas of faint or interrupted fluorescence in the distal portion of the filled fibers seen in the whole mount. Only a dark empty profile is seen in the section corresponding to the location in the whole mount where the dye-filled axon has ended (Fig. 4, $e$ and $f$ ). These empty profiles do not resemble the profiles of unfilled axons which have a detectable background fluorescence 


\section{Control}
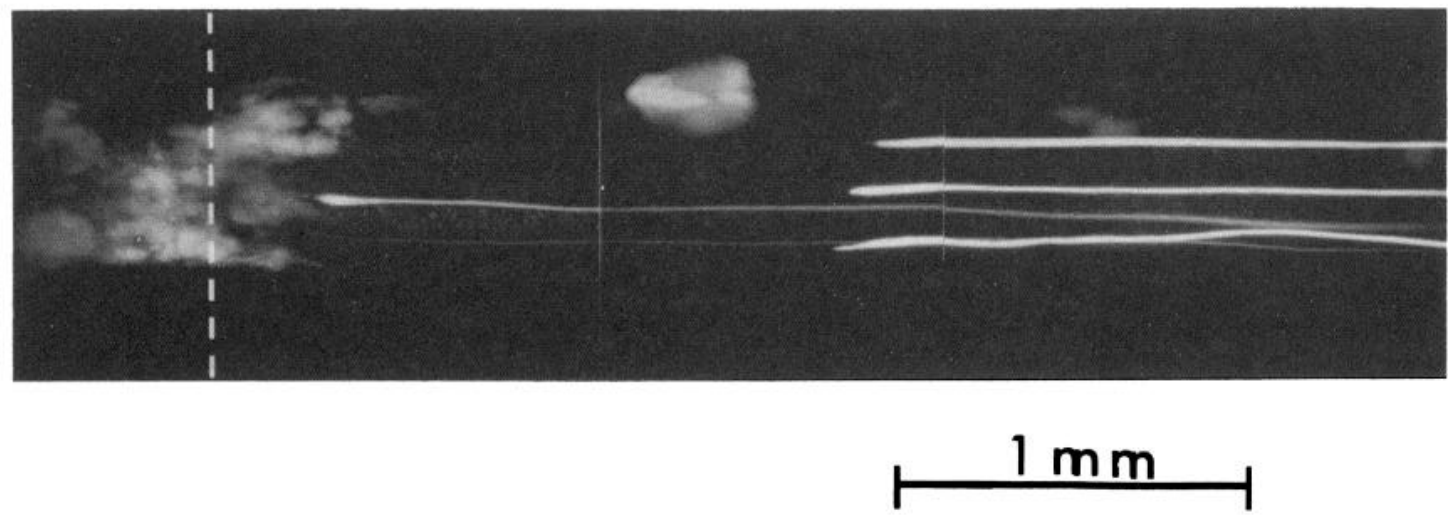

\section{Cathode Distal}

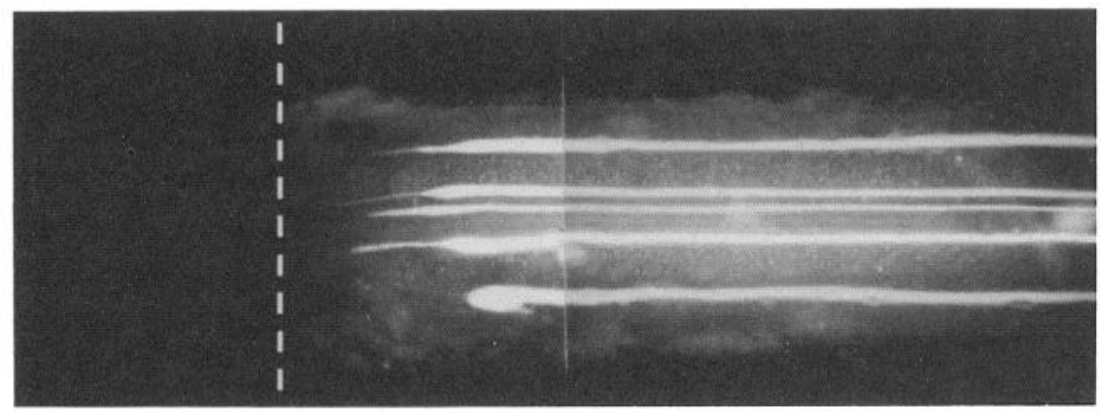

Figure 3. Montages of spinal cord whole mounts showing several giant axons filled with the fluorescent dye Lucifer Yellow 5 days after spinal transection. In each preparation the site of lesion is indicated by the dashed white line (rostral is to the right). Control, Electrodes were implanted but no current was passed. A total of four giant fibers were injected 5 days after lesion. The two most lateral are Mauthner axons, the two medial are Müller axons (cf. Fig. 2). Three of the giant fibers have died back from the lesion site approximately $1800 \mu \mathrm{m}$, while one remains within $250 \mu \mathrm{m}$ of the transection. Cathode Distal, Current was passed across the lesion for 5 days with the cathode distal to the site of transection. Then the axons were injected with fluorescent dye. Two Mauthner fibers (most lateral) and three Müller fibers have been injected. The fibers are within $550 \mu \mathrm{m}$ of the lesion or at the lesion itself.

(Fig. 4d). Therefore, we conclude that the terminal point of fluorescence in the whole mount represents the true end of the severed axon; it correlates with the end of fluorescent profiles and the beginning of black, empty spaces seen in the corresponding cross-sections.

Die-back distances in different treatment groups. A total of 107 giant axons in three experimental groups were filled with Lucifer Yellow. The distance of the axon end from the lesion was measured in the proximal stump 5 days after spinal cord transection. Of this total, 36 fibers were obtained from 10 sham-treated (control) preparations, 37 fibers were obtained from 8 cathode-distal preparations, and 34 fibers were obtained from 10 cathode-proximal preparations. The average distances of axon die-back were calculated for each experimental group and are shown in Figure 5 . It is apparent that 5

Figure 4. $a$, Montage of a spinal cord in whole mount viewed ventrally and showing four giant axons injected with dye 5 days after spinal transection. DC current was applied across the lesion (white dashed line) for 5 days after injury with the cathode proximal to the site of transection. The extent of axon die-back ranges from 650 to $5050 \mu \mathrm{m}$ and is greater than either in the control or in the cathode-distal preparation (cf. Fig. 3). Five-micrometer sections taken from the whole mount shown in $a$ are seen in $b$ to $f$ (ventral is upward). Black arrows from the whole mount to the sections indicate the approximate locations at which each section was taken. $b$, The two large, most lateral profiles are the injected Mauthner axons and correspond to the two lateral axons seen in $a$. A left Muller axon (curved arrow) and a right Müller axon (straight arrow) are seen as smaller bright oval profiles and correspond to the centrally located axons seen in $a$. Other Müller axons not injected with dye are visible due to background fluorescence. $c$, The curved arrow, which in $b$ marked the position of the filled left Müller cell, now points to a black empty profile and corresponds to the location of the whole mount where the left Müller cell is no longer visible. This profile is judged to be empty because it has the same density as the plastic outside of the cord and is much blacker than the profiles of unfilled fibers. $d$ and $e$, These two sections are taken from a region in the whole mount where the fluorescence is disappearing from the right Müller cell (straight arrow). Note the fragmented appearance of the fluorescence in $d$ and the partially empty black core appearing in $e$. $f$, The right Müller fiber is completely gone and is seen only as an empty, black profile (straight arrow). 

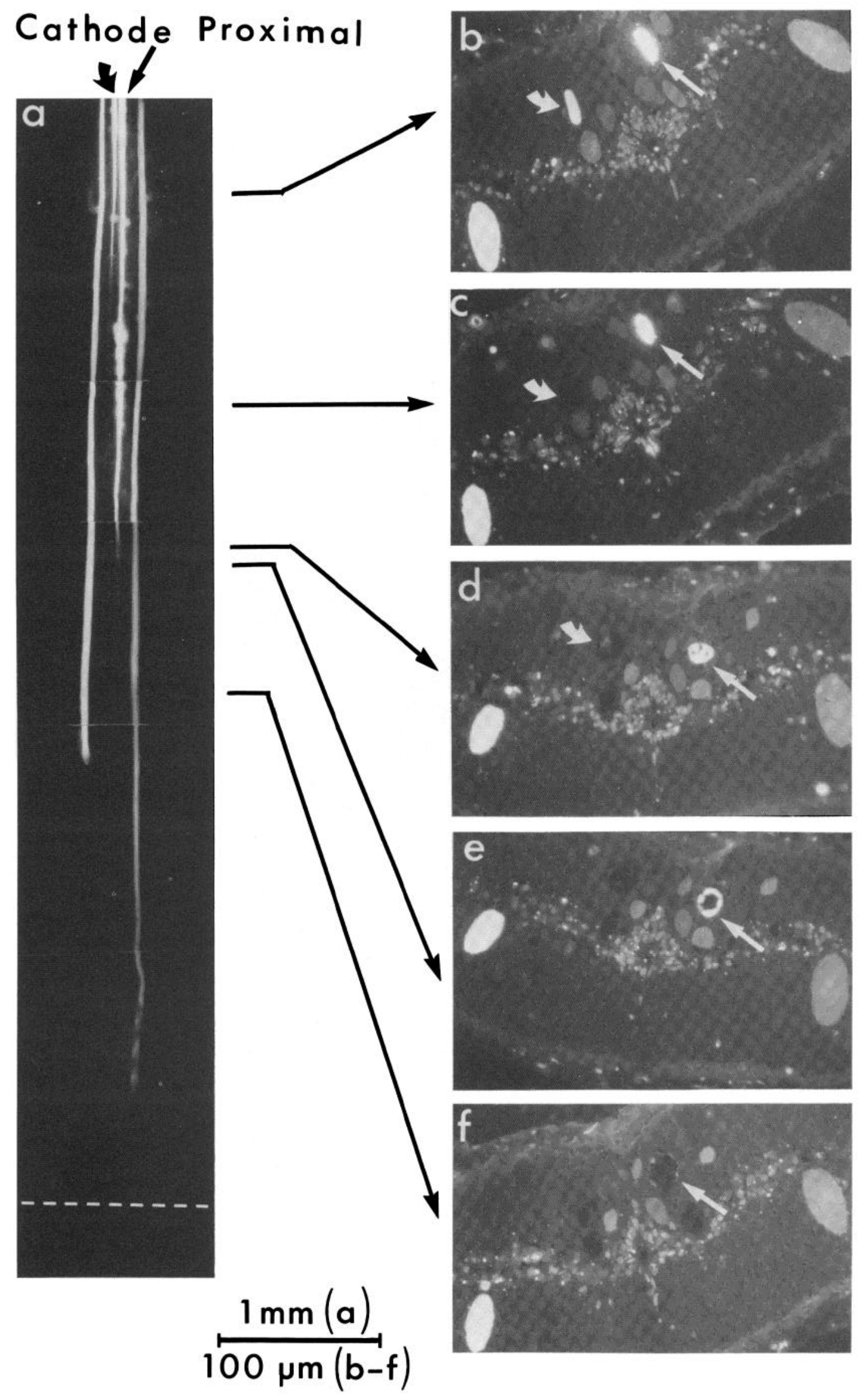

Figure 4 


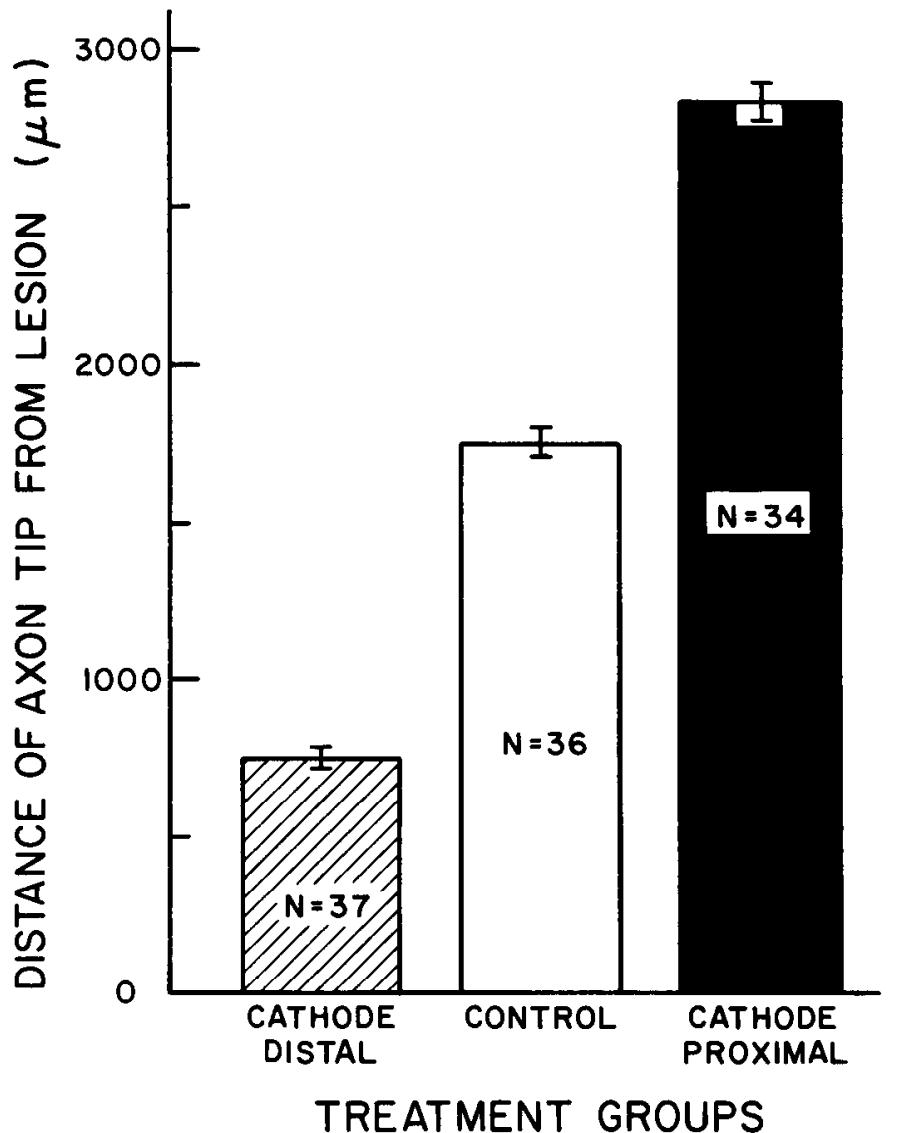

Figure 5. Diagram showing the mean and SEM of axon dieback in the three treatment groups. $N$ is the number of dyefilled fibers.

days of DC current across the lesion with the cathode distal to the injury site reduces the average extent of dieback (mean, $740 \mu \mathrm{m} \pm 33$ SEM) as compared to the controls (mean, $1750 \mu \mathrm{m} \pm 45$ SEM). Preparations treated with a cathode proximal to the lesion showed considerably greater die-back (mean, $2820 \mu \mathrm{m} \pm 60$ SEM). These differences in the mean distance of dieback in the different treatment groups were found to be statistically significant using the $\mathrm{Z}$ test $(p<0.01)$ (Peatman, 1963).

In order to examine the distribution of distances of the axon tips from the lesion in the three treatment groups, the data were ranked and plotted as a histogram in Figure 6. Clearly, the distribution of data from the two groups treated with DC current is different from that of the sham-treated control group. The measurements from the cathode-distal group are displaced closer to the lesion than are those of the sham-treated controls, while the data from the cathode-proximal group are displaced away from the lesion relative to the controls.

It is also evident from the histogram that the data from the cathode-distal group do not follow a normal distribution but are skewed toward the lesion. Therefore, because the data do not follow a normal distribution, the nonparametric Wilcoxon rank sum test (White, 1952) was used to determine if the differences seen between the three treatment groups were statistically significant. The results of this test are seen in the inset of Figure 6 and show that such differences are indeed significant.

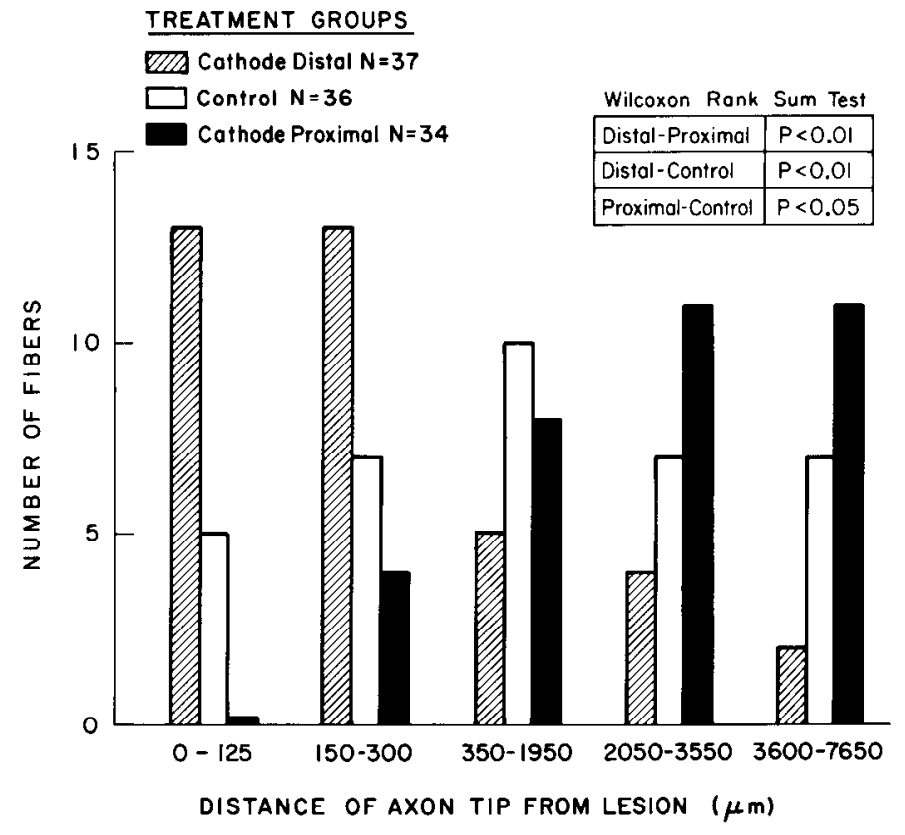

Figure 6. Distribution of the measured distances of the dyefilled axon ends from the lesion in the three treatment groups. The data were ranked according to increasing distance from the lesion. The histogram was drawn to have approximately the same total number of fibers plotted in each increment of distance. Note that the data from the cathode-distal group are skewed toward the lesion. The ranked data from the three pairs of treatment groups were compared by pairs using the Wilcoxon rank sum test. The results shown in the inset indicate that the differences between the three treatment groups are statistically significant.

\section{Discussion}

The giant axons of the reticulospinal neurons in the proximal segment of the transected lamprey spinal cord degenerate in a retrograde direction, i.e., die-back, for 1 to $2 \mathrm{~mm}$ from the site of injury by 5 days after lesion. We conclude in this study that DC current passed across the lesion, with the cathode distal, significantly reduces the die-back of giant axons 5 days after transection. DC current applied across the lesion in the opposite direction results in a significant enhancement of the die-back in the giant axons. A large and persistent injury current carried primarily by sodium and calcium has been shown to enter the cut proximal end of the lamprey spinal cord (Borgens et al., 1980). We postulate that the massive influx of calcium ions carried by the injury current into the cut end of the spinal cord is the primary agent that induces the die-back of severed axons in the proximal spinal cord segment. There are several additional lines of evidence indicating that calcium enters injured axons. In the injured mammalian spinal cord an increase in calcium in the area of lesion has been shown both by direct measurements (Happel et al., 1981) and by the appearance of accumulated masses of crystalline calcium in the parenchyma of the cord (Banik et al., 1980). An increased conductance to calcium has been shown at the cut end of giant interneurons in the cockroach (Meiri et al., 1981), thus suggesting that the intracellular calcium level increases in these central neurons as well following axotomy. 
The critical role of intracellular calcium in maintaining the integrity of the cytoskeleton is well documented. It has long been known that a high level of intracellular calcium results directly in a depolymerization of microtubules (Wilson et al., 1970; Borisy and Olmsted, 1972; Weisenberg, 1972; Kirschner and Williams, 1974). Such an increased intracellular calcium level also causes breakdown of neurofilaments by activating a calcium-dependent protease (Gilbert et al., 1975; Pant et al., 1979). In addition, the manipulation of calcium in the external medium has been shown to modify the extent of Wallerian degeneration in isolated axonal segments (Schlaepfer and Bunge, 1973; Schlaepfer, 1974). Lowering the calcium concentration in the external medium by the substitution of magnesium for calcium or the addition of EGTA as a calcium chelating agent prevents or delays the breakdown of isolated axonal segments in tissue culture. Conversely, the addition of excess calcium to the external medium accelerates the degeneration of isolated axonal segments. Schlaepfer (1974) has proposed that a large influx of calcium into the injured axon and its role in the disassembly of fibrous cytoskeletal elements
(Schlaepfer, 1977; Schlaepfer and Hasler, 1979) is the critical event leading to Wallerian degeneration of isolated distal axonal segments.

We propose that a similar influx of calcium into the proximal end of the injured spinal cord, via the injury current, results in the observed axonal die-back seen in the control preparations. Thus, any factor which modifies the amount of current entering the transected cord will modify the amount of calcium flowing into the cord and therefore affect the extent of die-back in the injured proximal axon segments. The applied DC current described in this study then could interact with the current of injury such that the net amount of current flowing into the injured cord is modified. If the cathode is oriented distal to the site of lesion, the applied current counteracts the endogenous injury current resulting in a decrease of the net current entering the cut end of the axon as shown in Figure 7. This decreases the total amount of calcium that enters these axons and thus reduces the extent of die-back. If the cathode delivering the applied DC current is oriented proximal to the site of lesion, then the endogenous and exogenous currents will
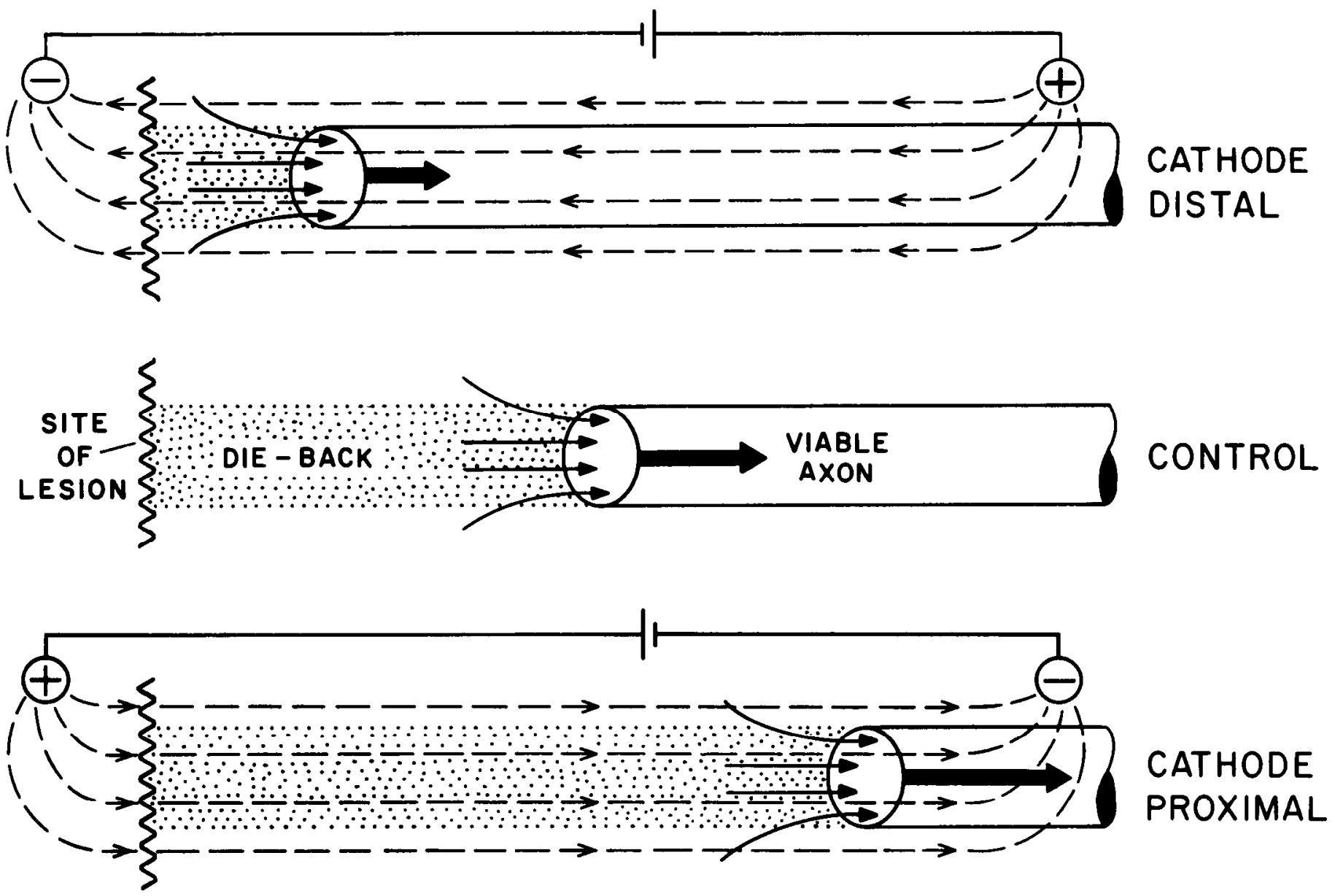

Figure 7. A summary diagram indicating the proposed interaction between endogenous injury current and applied electric current, showing the extent of die-back for a single giant axon in each treatment group. We postulate that the net inward current (single broad solid arrow) causes the die-back seen in each of the three treatment groups. In the sham-treated controls, the only DC current entering the axon is from the endogenous injury current(solid thin arrows) and this gives rise to the intermediate range of axonal die-back seen in this group. In the cathode-proximal group, the applied DC current (dashed lines) sums with the endogenous injury current to produce an increase in the total inward current entering the cut axon face with respect to the control group. This could explain the observed increase in the extent of die-back seen in this group as compared to the controls. On the other hand, in the cathode-distal group, the applied current opposes the endogenous injury current to produce a decrease in the net inward current. This causes a decrease in the extent of die-back as compared to the other two groups. 
sum, causing an increase of the net current entering the cut face of the axon. This results in an even greater influx of calcium into the injured axon and thus increases the extent of axon die-back when compared to either the controls or the cathode-distal treatment group (Fig. 7).

It is known that applied DC current with the cathode distal to the lesion enhances regeneration of the giant axons in the lamprey spinal cord 40 to 60 days after transection (Borgens et al., 1981). We propose that this enhancement of regeneration is related to the early modification of the die-back reported here. We show that applied DC current, with the cathode oriented distal to the lesion, will diminish the extent of axon die-back. This means that after 5 days of treatment, the ends of giant axons in these animals generally are closer to the site of lesion than in untreated animals. Hence, if growth begins al some fixed lime after injury, then the axons in the cathode-distal treatment group would reach the lesion area earlier than in controls because axon outgrowth starts closer to the lesion. This is precisely what was observed 40 to 60 days after injury in our previous study (Borgens et al., 1981) at which time more axons were in or across the site of lesion in the group treated with DC current than in the sham-treated controls.

In summary, we have shown that application of a DC current across a spinal lesion can either enhance or reduce the extent of die-back in proximal axon stumps, depending on the orientation of the applied current. We postulate that this occurs by an interaction between the applied DC current and the endogenous injury current. This, in turn, determines the net influx of calcium into the cut end of the cord. The total influx of calcium may then influence the cxtent of retrograde degeneration (dieback) of proximal axonal segments by affecting the stability of the fibrous proteins comprising the neuronal cytoskeleton. The amount of die-back then can affect the course of subsequent regeneration by determining the distance from the lesion at which the regenerating growth begins.

\section{References}

Banik, N. L., J. M. Powers, and E. L. Hogan (1980) The effects of spinal cord trauma on myelin. J. Neuropathol. Exp. Neurol. 39: 232-244.

Borgens, R. B., L. F. Jaffe, and M. J. Cohen (1980) Large and persistent currents enter the transected lamprey spinal cord. Proc. Natl. Acad. Sci. U. S. A. 77: 1209-1213.

Borgens, R. B., E. Roederer, and M. J. Cohen (1981) Enhanced spinal cord regeneration in lamprey by applied electric fields. Science 213: 611-617.

Borisy, G. G., and J. B. Olmsted (1972) Nucleated assembly of microtubules in porcine brain extracts. Science 177: 1196-1197.
Cajal, S. Ramon y (1928) Degeneration and Regeneration of the Nervous System, Vol. 2, pp. 487-516, Oxford University Press, London.

Friede, R. L., and A. J. Martinez (1970) Analysis of the process of sheath expansion in swollen nerve fibers. Brain Res. 19: $165-182$.

Gilbert, D. S., B. J. Newby, and B. H. Anderton (1975) Neurofilament disguise, destruction and discipline. Nature 256: 586-589.

Happel, R. D., K. P. Smith, N. L. Banik, J. M. Powers, E. L. Hogan, and J. D. Balentine (1981) $\mathrm{Ca}^{2+}$-accumulation in experimental spinal cord trauma. Brain Res. 211: 476-479.

Kao, C. C., and L. W. Chang (1977) The mechanism of spinal cord cavitation following spinal cord transection. J. Neurosurg. 46: 197-209.

Kao, C. C., L. W. Chang, and J. M. B. Bloodworth, Jr. (1977) Electron microscopic observations of the mechanisms of terminal club formation in transected spinal cord axons. J. Neuropathol. Exp. Neurol. 36: 140-156.

Kirschner, M. W., and R. C. Williams (1974) The mechanism of microtubule assembly in vitro. J. Supramol. Struct. 2: 412-428.

Lasek, R. J. (1981) The dynamic ordering of neuronal cytoskeletons. Neurosci. Res. Program Bull. 19: 7-32.

Meiri, H., M. E. Spira, and I. Parnas (1981) Membrane conductance and action potential of a regenerating axonal tip. Science 211: 709-712.

Pant, H. C., S. Terakawa, and H. Gainer (1979) A calcium activated protease in squid axoplasm. J. Neurochem. 32: 99-102.

Peatment, J. G. (1963) Introduction to Applied Statistics. Harper and Row, New York.

Schlaepfer, W. W. (1974) Calcium-induced degeneration of axoplasm in isolated segments of rat peripheral nerve. Brain Res. 69: 203-215.

Schlaepfer, W. W. (1977) Structural alterations of peripheral nerve induced by the calcium ionophore A23187. Brain Res. 136: 1-9.

Schlaepfer, W. W., and R. P. Bunge (1973) Effects of calcium ion concentration on the degeneration of amputated axons in tissue culture. J. Cell. Biol. 59: 456-470.

Schlaepfer, W. W., and M. B. Hasler (1979) Characterization of the calcium-induced disruption of neurofilaments in rat peripheral nerve. Brain Res. 168: 299-309.

Stewart, W. W. (1978) Functional connections between cells as revealed by dye-coupling with a highly fluorescent naphthalimide tracer. Cell 14: 741-759.

Weisenberg, R. C. (1972) Microtubule formation in vitro in solutions containing low calcium concentrations. Science 177: 1104-1105.

White, C. (1952) The use of ranks in a test of significance for comparing two treatments. Biometrics $8: 33-41$.

Wickelgren, W. O. (1977) Physiological and anatomical characteristics of reticulospinal neurones in lamprey. J. Physiol. (Lond.) 270: 89-114.

Wilson, L., J. Bryan, A. Ruby, and D. Mazia (1970) Precipitation of proteins by vinblastine and calcium ions. Proc. Natl. Acad. Sci. U. S. A. 66: 807-814. 